\title{
Double diastolic murmur in mitral stenosis with atrial fibrillation and complete heart block
}

\author{
PAVLOS TOUTOUZAS, ANASTASIOS VELIMEZIS, \\ DEMETRIS AVGOUSTAKIS
}

From Department of Cardiology, University of Athens Medical School, Hippokration Hospital, Athens, Greece

SUMMARY A double diastolic murmur was heard in a patient with mitral stenosis, atrial fibrillation, and complete heart block. Echo-phonocardiographic examination showed two separate opening and closing movements of the mitral valve in the same long diastole. A clear time relation was observed between the valve closing movements and the separate diastolic murmurs, giving support to the theory that the backward motion of the mitral valve against the forward blood flow through the valve is responsible for the production of these murmurs.

A few years ago, a new theory of the genesis of the mitral diastolic murmur was proposed, namely that the murmur resulted from impact between the forward mitral flow and the closing motion of the mitral valve (Criley et al., 1971; Fortuin and Craige, 1973; Toutouzas et al., 1974). This theory was challenged recently in an editorial by Tavel and Bonner (1976).

We are now reporting the unusual case of a patient with mitral stenosis, atrial fibrillation, and complete heart block, who had a double diastolic murmur, and in whom the role of the closing motion of the mitral valve in the genesis of the murmur was clearly demonstrated.

\section{Case report}

A 59-year-old woman entered hospital in September 1975 with a 2-month history of dizziness and syncopal attacks. She was not hypertensive and had never complained of dyspnoea or other symptoms of heart failure. Complete heart block was diagnosed

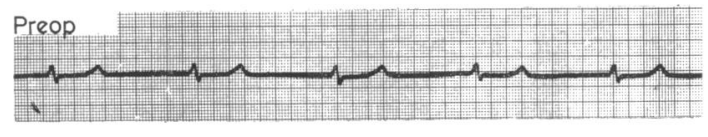

Lead II

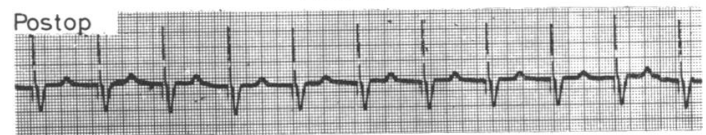

Fig. 1 Lead II of the electrocardiogram before and during pacing. There are no $P$ waves in either record.
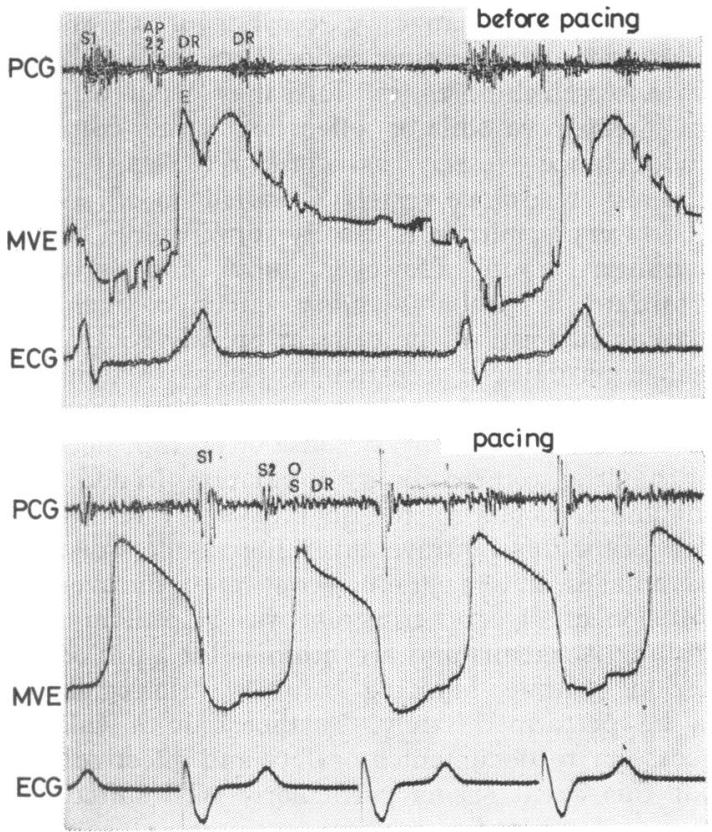

Fig. 2 Simultaneous recordings of the phonocardiogram (PCG), mitral valve echogram (MVE), and electrocardiogram (ECG). Above: Complete heart block and atrial fibrillation. The PCG shows two rumbling murmurs $(D R)$ in the same long diastole. The $M V E$ shows a second opening motion of the valve after the first diastolic closure slope. Note the time relation between the two murmurs and closing motions of the mitral valve. Below: After implantation of pacemaker. PCG and $M V E$ show typical features of mitral stenosis (S1: 1st heart sound, S2: 2nd heart sound, OS: opening snap). 


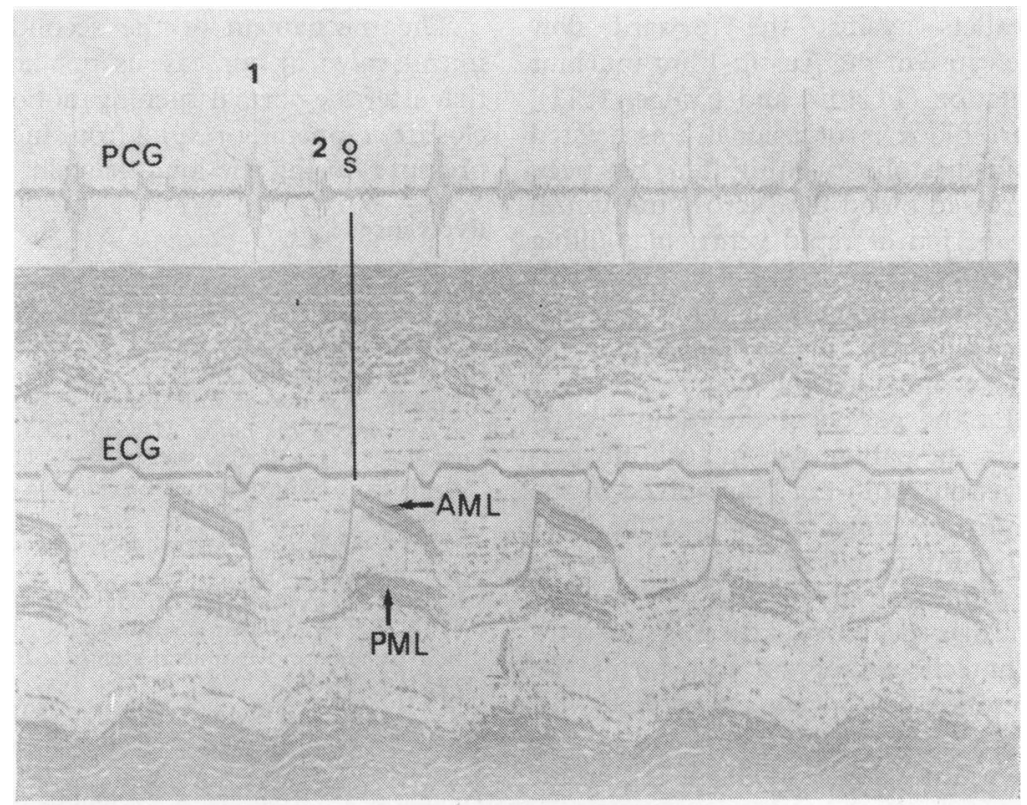

Fig. 3 Recent echocardiogram with simultaneous phonocardiogram (PCG) and ECG lead II during pacing. The mitral valve echogram shows typical changes of mitral stenosis with anterior motion of the posterior mitral leaflet $(P M L)$. The PCG shows a distinct opening snap (OS), coinciding with the end of the rapid anterior mitral leaflet (AML) upstroke, and a diastolic murmur coinciding with the diastolic closure slope.

on the clinical examination, and the electrocardiogram showed atrial fibrillation with an idioventricular rhythm at a rate of 35 per minute (Fig. 1). On $x$-ray examination the heart was moderately enlarged.

The clinical features of heart block were associated with an interesting auscultatory sign, a double diastolic rumbling murmur at the apex. This was confirmed by a phonocardiogram showing two separate murmurs during the same long diastole. The echocardiogram showed two distinct opening and closing movements of the mitral valve corresponding with the separate diastolic murmurs. Subsequently, a combined echo-phonocardiographic examination was carried out; the gated mitral valve signal from the Hewlett-Packard ultrasound device was recorded simultaneously with the phonocardiogram using an Electronics for Medicine multichannel recorder. We observed a clear time relation between the two closing motions of the mitral valve and the diastolic murmurs (Fig. 2). The patient was treated by a pacemaker implantation for the heart block. At a heart rate of 70 beats per minute, an opening snap and a single diastolic murmur were heard. The postoperative echo-phonocardiographic examination showed the typical pattern of mitral stenosis, with anterior motion of the posterior mitral leaflet, and again a time relation between the diastolic murmur and diastolic closure motion on the echocardiogram (Fig. 3).

\section{Discussion}

According to the new theory the mitral diastolic murmurs occur as the result of an impact between increased velocity of forward blood flow and the backward motion of the mitral valve (Fortuin and Craige, 1973; Toutouzas et al., 1974). This theory explains some features of the diastolic murmurs in mitral stenosis, such as the absence of murmur before and a few milliseconds after the opening snap (Toutouzas et al., 1974, 1978), the occasional presence of a presystolic murmur in atrial fibrillation (Criley and Hermer, 1971; Lakier et al., 1972; Toutouzas et al., 1974), and the low intensity or absence of the murmur in mitral stenosis with a calcified mitral valve. In addition, this theory accounts for the appearance of mid-diastolic murmurs well after the period of rapid filling in patients with mitral regurgitation or with congenital heart disease and a large left-to-right shunt (Fortuin and Craige, 1973). Finally, the closing motion of the 
mitral valve leaflets against the forward flow appears to be the cause of the Austin-Flint murmur in aortic regurgitation (Fortuin and Craige, 1972).

Complete heart block is occasionally associated with an apical mid-diastolic murmur. This has been attributed to increased blood flow across the mitral valve during the period of rapid ventricular filling and is louder when atrial contraction occurs at this time. However, in our patient with mitral stenosis and complete heart block, this explanation is insufficient. The second diastolic murmur was recorded well after the period of the rapid ventricular filling and, as the patient was in atrial fibrillation, atrial contraction could not be involved in the genesis of the murmur. Fig. 2 illustrates the clear time relation of the closing motion of the valve to the two separate murmurs observed during the same long diastole. The disappearance of the murmur after the $F$ point of the echocardiogram and during the second opening motion of the valve, combined with reappearance of the murmur with the second closing valve motion, leaves little doubt about the important role of closing motion of the valve in its genesis. Thus, we find it very difficult to accept the theory of Tavel and Bonner (1976) who state that the diastolic murmur does not appear during the opening valve motions, 'since the mitral apparatus is moving forward with the atrioventricular blood during these periods', reducing mitral flow and turbulence.
The mechanism of the second opening of the mitral valve in our case is not known. It is likely that after the normal opening motion and subsequent closure, reopening results from increasing left atrial pressure during the long diastole.

\section{References}

Criley, J. M., Feldmar, I. M., and Meredit, T. (1971). Mitral valve closure and the crescendo presystolic murmur. American fournal of Medicine, 51, 456-465.

Criley, J. M., and Hermer, A. J. (1971). The crescendo presystolic murmur of mitral stenosis with atrial fibrillation. New England fournal of Medicine, 285, 1284-1287.

Fortuin, N. J., and Craige, E. (1972). On the mechanism of the Austin-Flint murmur. Circulation, 45, 558-570.

Fortuin, N. J., and Craige, E. (1973). Echocardiographic studies of genesis of mitral diastolic murmur. British Heart fournal, 35, 75-81.

Lakier, J. B., Pocock, W. A., Gale, G. E., and Barlow, J. B. (1972). Haemodynamic and sound events preceding the first heart sound in mitral stenosis. British Heart fournal, 34, 1152-1155.

Tavel, M. E., and Bonner, A. J., jun (1976). Presystolic murmur in atrial fibrillation: fact or fiction? Circulation, 54, 167-168.

Toutouzas, P., Koidakis, A., Velimezis, A., and Avgoustakis, D. (1974). Mechanism of diastolic rumble and presystolic murmur in mitral stenosis. British Heart fournal, 36, 10961105.

Toutouzas, P., Velimezis, A., Kastellanos, S., and Avgoustakis, D. (1978). Non-invasive study of early diastole in mitral stenosis. Circulation, 57, 708-715.

Requests for reprints to Dr Pavlos Toutouzas, 10 Likiou str., Athens 138, Greece. 